\title{
Highlight report: xenobiotic-metabolizing enzymes in skin models
}

\author{
Klaus Golka ${ }^{1}$
}

Published online: 1 December 2015

(C) Springer-Verlag Berlin Heidelberg 2015

Recently, Franz Oesch and colleagues have published a comprehensive review about the xenobiotic-metabolizing activity in skin of rat, mouse, pig, guinea pig, man, and human skin models (Oesch et al. 2014). Since the skin is constantly exposed to different xenobiotics, its xenobiotic-metabolizing activity is of high relevance. The use of human skin is limited for ethical and practical reasons. Therefore, non-human skin and in vitro skin models are frequently applied (Oesch et al. 2014). The authors give a comprehensive overview over possibilities and limitations of the most frequently applied in vitro systems used in skin toxicology. Some critical key messages are as follows:

- For xenobiotic metabolism relevant for dermal absorption, the pig skin most closely resembles the human skin compared to the other analyzed species. Similarly, for studies of skin irritation, the pig skin represents an adequate model for humans.

- Moreover, some reconstructed human skin models are reasonably close to primary human skin.

- However, currently available skin models have a too low barrier function to correctly estimate dermal absorption.

- With respect to genotoxicity and sensitization, activating metabolism has not yet been sufficiently studied to come to final conclusions.

In vitro systems of skin metabolism and absorption represent a cutting-edge topic in toxicology (Manwaring et al.

Klaus Golka

golka@ifado.de

1 Leibniz Research Centre for Working Environment and Human Factors at TU Dortmund, IfADo, Ardeystr. 67, 44139 Dortmund, Germany
2015; Jacquoilleot et al. 2015; Crane et al. 2015; Gegotek and Skrzydlewska 2015; Manevski et al. 2015; Wiegand et al. 2014). As for other in vitro systems, e.g., for liver (Reif et al. 2015; Godoy et al. 2013, 2015; Watzek et al. 2013; Schug et al. 2013; Fang et al. 2014; Grinberg et al. 2014), kidney (Sanchez-Niño et al. 2014; Fujiki et al. 2014, 2013), and neuronal system (Balmer et al. 2014; Zimmer et al. 2014; Krug et al. 2013; Waldmann et al. 2014), it is important to know the metabolizing capacity in relation to the in vivo situation. A model that represents the human situation in all aspects will possibly never be accomplished (Ghallab 2013, 2014a, b; Stewart 2010). One limitation is that it is extremely difficult to sufficiently maintain all critical biochemical factors of the in vivo microenvironment, when cells are isolated from an organism and transferred to artificial culture conditions (Godoy et al. 2009, 2010a, b; Godoy 2011). Therefore, Oesch et al. (2014) have chosen a reasonable strategy in this complex situation: Carefully scrutinize the properties of the available in vitro systems to allow an informed decision about endpoints which can be reliably studied because of sufficient similarity to the human in vivo situation.

\section{References}

Balmer NV, Klima S, Rempel E, Ivanova VN, Kolde R, Weng MK, Meganathan K, Henry M, Sachinidis A, Berthold MR, Hengstler JG, Rahnenführer J, Waldmann T, Leist M (2014) From transient transcriptome responses to disturbed neurodevelopment: role of histone acetylation and methylation as epigenetic switch between reversible and irreversible drug effects. Arch Toxicol 88(7):1451-1468. doi:10.1007/s00204-014-1279-6

Crane JD, MacNeil LG, Lally JS, Ford RJ, Bujak AL, Brar IK, Kemp BE, Raha S, Steinberg GR, Tarnopolsky MA (2015) Exercise-stimulated interleukin-15 is controlled by AMPK and 
regulates skin metabolism and aging. Aging Cell 14(4):625-634. doi:10.1111/acel.12341

Fang JL, Han T, Wu Q, Beland FA, Chang CW, Guo L, Fuscoe JC (2014) Differential gene expression in human hepatocyte cell lines exposed to the antiretroviral agent zidovudine. Arch Toxicol 88(3):609-623. doi:10.1007/s00204-013-1169-3

Fujiki K, Inamura H, Matsuoka M (2013) Phosphorylation of FOXO3a by PI3K/Akt pathway in HK-2 renal proximal tubular epithelial cells exposed to cadmium. Arch Toxicol 87(12):21192127. doi:10.1007/s00204-013-1077-6

Fujiki K, Inamura H, Matsuoka M (2014) PI3K signaling mediates diverse regulation of ATF4 expression for the survival of HK-2 cells exposed to cadmium. Arch Toxicol 88(2):403-414. doi:10.1007/s00204-013-1129-y

Gęgotek A, Skrzydlewska E (2015) The role of transcription factor Nrf2 in skin cells metabolism. Arch Dermatol Res 307(5):385396. doi:10.1007/s00403-015-1554-2

Ghallab A (2013) In vitro test systems and their limitations. EXCLI J 12:1024-1026

Ghallab A (2014a) Human non-parenchymal liver cells for co-cultivation systems. EXCLI J 13:1295-1296

Ghallab A (2014b) The rediscovery of HepG2 cells for prediction of drug induced liver injury (DILI). EXCLI J 13:1286-1288

Godoy P (2011) Hepatotoxicity. EXCLI J 10:124-127

Godoy P, Hengstler JG, Ilkavets I, Meyer C, Bachmann A, Müller A, Tuschl G, Mueller SO, Dooley S (2009) Extracellular matrix modulates sensitivity of hepatocytes to fibroblastoid dedifferentiation and transforming growth factor beta-induced apoptosis. Hepatology 49(6):2031-2043. doi:10.1002/hep.22880

Godoy P, Schug M, Bauer A, Hengstler JG (2010a) Reversible manipulation of apoptosis sensitivity in cultured hepatocytes by matrix-mediated manipulation of signaling activities. Methods Mol Biol 640:139-155. doi:10.1007/978-1-60761-688-7_7

Godoy P, Lakkapamu S, Schug M, Bauer A, Stewart JD, Bedawi E, Hammad S, Amin J, Marchan R, Schormann W, Maccoux L, von Recklinghausen I, Reif R, Hengstler JG (2010b) Dexamethasone-dependent versus-independent markers of epithelial to mesenchymal transition in primary hepatocytes. Biol Chem 391(1):73-83. doi:10.1515/BC.2010.010

Godoy P, Hewitt NJ, Albrecht U, Andersen ME, Ansari N, Bhattacharya S, Bode JG, Bolleyn J, Borner C, Böttger J, Braeuning A, Budinsky RA, Burkhardt B, Cameron NR, Camussi G, Cho CS, Choi YJ, Craig Rowlands J, Dahmen U, Damm G, Dirsch O, Donato MT, Dong J, Dooley S, Drasdo D, Eakins R, Ferreira KS, Fonsato V, Fraczek J, Gebhardt R, Gibson A, Glanemann M, Goldring CE, Gómez-Lechón MJ, Groothuis GM, Gustavsson L, Guyot C, Hallifax D, Hammad S, Hayward A, Häussinger D, Hellerbrand C, Hewitt P, Hoehme S, Holzhütter HG, Houston JB, Hrach J, Ito K, Jaeschke H, Keitel V, Kelm JM, Kevin Park B, Kordes C, Kullak-Ublick GA, LeCluyse EL, Lu P, Luebke-Wheeler J, Lutz A, Maltman DJ, Matz-Soja M, McMullen P, Merfort I, Messner S, Meyer C, Mwinyi J, Naisbitt DJ, Nussler AK, Olinga P, Pampaloni F, Pi J, Pluta L, Przyborski SA, Ramachandran A, Rogiers V, Rowe C, Schelcher C, Schmich K, Schwarz M, Singh B, Stelzer EH, Stieger B, Stöber R, Sugiyama Y, Tetta C, Thasler WE, Vanhaecke T, Vinken M, Weiss TS, Widera A, Woods CG, Xu JJ, Yarborough KM, Hengstler JG (2013) Recent advances in 2D and 3D in vitro systems using primary hepatocytes, alternative hepatocyte sources and non-parenchymal liver cells and their use in investigating mechanisms of hepatotoxicity, cell signaling and ADME. Arch Toxicol 87(8):1315-1530

Godoy P, Schmidt-Heck W, Natarajan K, Lucendo-Villarin B, Szkolnicka D, Asplund A, Björquist P, Widera A, Stöber R, Campos G, Hammad S, Sachinidis A, Chaudhari U, Damm G, Weiss TS, Nüssler A, Synnergren J, Edlund K, Küppers-Munther B,
Hay DC, Hengstler JG (2015) Gene networks and transcription factor motifs defining the differentiation of stem cells into hepatocyte-like cells. J Hepatol 63(4):934-942. doi:10.1016/j. jhep.2015.05.013

Grinberg M, Stöber RM, Edlund K, Rempel E, Godoy P, Reif R, Widera A, Madjar K, Schmidt-Heck W, Marchan R, Sachinidis A, Spitkovsky D, Hescheler J, Carmo H, Arbo MD, van de Water B, Wink S, Vinken M, Rogiers V, Escher S, Hardy B, Mitic D, Myatt G, Waldmann T, Mardinoglu A, Damm G, Seehofer D, Nüssler A, Weiss TS, Oberemm A, Lampen A, Schaap MM, Luijten M, van Steeg H, Thasler WE, Kleinjans JC, Stierum RH, Leist M, Rahnenführer J, Hengstler JG (2014) Toxicogenomics directory of chemically exposed human hepatocytes. Arch Toxicol 88(12):2261-2287. doi:10.1007/s00204-014-1400-x

Jacquoilleot S, Sheffield D, Olayanju A, Sison-Young R, Kitteringham NR, Naisbitt DJ, Aleksic M (2015) Glutathione metabolism in the HaCaT cell line as a model for the detoxification of the model sensitisers 2,4-dinitrohalobenzenes in human skin. Toxicol Lett 237(1):11-20. doi:10.1016/j.toxlet.2015.05.016

Krug AK, Kolde R, Gaspar JA, Rempel E, Balmer NV, Meganathan K, Vojnits K, Baquié M, Waldmann T, Ensenat-Waser R, Jagtap S, Evans RM, Julien S, Peterson H, Zagoura D, Kadereit S, Gerhard D, Sotiriadou I, Heke M, Natarajan K, Henry M, Winkler J, Marchan R, Stoppini L, Bosgra S, Westerhout J, Verwei M, Vilo J, Kortenkamp A, Hescheler J, Hothorn L, Bremer S, van Thriel C, Krause KH, Hengstler JG, Rahnenführer J, Leist M, Sachinidis A (2013) Human embryonic stem cell-derived test systems for developmental neurotoxicity: a transcriptomics approach. Arch Toxicol 87(1):123-143. doi:10.1007/s00204-012-0967-3

Manevski N, Swart P, Balavenkatraman KK, Bertschi B, Camenisch G, Kretz O, Schiller H, Walles M, Ling B, Wettstein R, Schaefer DJ, Itin P, Ashton-Chess J, Pognan F, Wolf A, Litherland K (2015) Phase II metabolism in human skin: skin explants show full coverage for glucuronidation, sulfation, $\mathrm{N}$-acetylation, catechol methylation, and glutathione conjugation. Drug Metab Dispos 43(1):126-139. doi:10.1124/dmd.114.060350

Manwaring J, Rothe H, Obringer C, Foltz DJ, Baker TR, Troutman JA, Hewitt NJ, Goebel C (2015) Extrapolation of systemic bioavailability assessing skin absorption and epidermal and hepatic metabolism of aromatic amine hair dyes in vitro. Toxicol Appl Pharmacol 287(2):139-148. doi:10.1016/j.taap.2015.05.016

Oesch F, Fabian E, Guth K, Landsiedel R (2014) Xenobiotic-metabolizing enzymes in the skin of rat, mouse, pig, guinea pig, man, and in human skin models. Arch Toxicol 88:2135-2190. doi:10.1007/s00204-014-1382-8

Reif R, Karlsson J, Günther G, Beattie L, Wrangborg D, Hammad S, Begher-Tibbe B, Vartak A, Melega S, Kaye PM, Hengstler JG, Jirstrand M (2015) Bile canalicular dynamics in hepatocyte sandwich cultures. Arch Toxicol 89(10):1861-1870. doi:10.1007/ s00204-015-1575-9

Sanchez-Niño MD, Poveda J, Sanz AB, Carrasco S, Ruiz-Ortega M, Selgas R, Egido J, Ortiz A (2014) 3,4-DGE is cytotoxic and decreases HSP27/HSPB1 in podocytes. Arch Toxicol 88(3):597608. doi:10.1007/s00204-013-1181-7

Schug M, Stöber R, Heise T, Mielke H, Gundert-Remy U, Godoy P, Reif R, Blaszkewicz M, Ellinger-Ziegelbauer H, Ahr HJ, Selinski S, Günther G, Marchan R, Blaszkewicz M, Sachinidis A, Nüssler A, Oberemm A, Hengstler JG (2013) Pharmacokinetics explain in vivo/in vitro discrepancies of carcinogen-induced gene expression alterations in rat liver and cultivated hepatocytes. Arch Toxicol 87(2):337-345. doi:10.1007/s00204-012-0999-8

Stewart JD (2010) In vitro test systems in toxicology. EXCLI J 9:156-158

Waldmann T, Rempel E, Balmer NV, König A, Kolde R, Gaspar JA, Henry M, Hescheler J, Sachinidis A, Rahnenführer J, Hengstler JG, Leist M (2014) Design principles of concentration-dependent 
transcriptome deviations in drug-exposed differentiating stem cells. Chem Res Toxicol 27(3):408-420. doi:10.1021/tx400402j

Watzek N, Scherbl D, Schug M, Hengstler JG, Baum M, Habermeyer M, Richling E, Eisenbrand G (2013) Toxicokinetics of acrylamide in primary rat hepatocytes: coupling to glutathione is faster than conversion to glycidamide. Arch Toxicol 87(8):1545-1556. doi:10.1007/s00204-013-1054-0

Wiegand C, Hewitt NJ, Merk HF, Reisinger K (2014) Dermal xenobiotic metabolism: a comparison between native human skin, four in vitro skin test systems and a liver system. Skin Pharmacol Physiol 27(5):263-275. doi:10.1159/000358272

Zimmer B, Pallocca G, Dreser N, Foerster S, Waldmann T, Westerhout J, Julien S, Krause KH, van Thriel C, Hengstler JG, Sachinidis A, Bosgra S, Leist M (2014) Profiling of drugs and environmental chemicals for functional impairment of neural crest migration in a novel stem cell-based test battery. Arch Toxicol 88(5):1109-1126. doi:10.1007/s00204-014-1231-9 